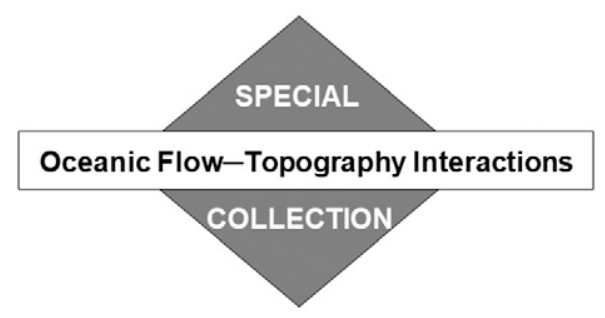

\title{
○Parameterizing Nonpropagating Form Drag over Rough Bathymetry
}

\author{
Jody M. Klymak, ${ }^{a}$ Dhruv Balwada, ${ }^{\mathrm{b}}$ Alberto Naveira Garabato, ${ }^{\mathrm{c}}$ And Ryan Abernathey ${ }^{\mathrm{d}}$ \\ ${ }^{\text {a }}$ University of Victoria, Victoria, British Columbia, Canada \\ ${ }^{\mathrm{b}}$ University of Washington, Seattle, Washington \\ ${ }^{\mathrm{c}}$ National Oceanography Centre, University of Southampton, Southampton, United Kingdom \\ ${ }^{\mathrm{d}}$ Columbia University, New York City, New York
}

(Manuscript received 21 May 2020, in final form 3 February 2021)

\begin{abstract}
Slowly evolving stratified flow over rough topography is subject to substantial drag due to internal motions, but often numerical simulations are carried out at resolutions where this "wave" drag must be parameterized. Here we highlight the importance of internal drag from topography with scales that cannot radiate internal waves, but may be highly nonlinear, and we propose a simple parameterization of this drag that has a minimum of fit parameters compared to existing schemes. The parameterization smoothly transitions from a quadratic drag law $\left(\sim h u_{0}^{2}\right)$ for low $N h / u_{0}($ linear wave dynamics) to a linear drag law $\left(\sim h^{2} u_{0} N\right)$ for high $N h / u_{0}$ flows (nonlinear blocking and hydraulic dynamics), where $N$ is the stratification, $h$ is the height of the topography, and $u_{0}$ is the near-bottom velocity; the parameterization does not have a dependence on Coriolis frequency. Simulations carried out in a channel with synthetic bathymetry and steady body forcing indicate that this parameterization accurately predicts drag across a broad range of forcing parameters when the effect of reduced nearbottom mixing is taken into account by reducing the effective height of the topography. The parameterization is also tested in simulations of wind-driven channel flows that generate mesoscale eddy fields, a setup where the downstream transport is sensitive to the bottom drag parameterization and its effect on the eddies. In these simulations, the parameterization replicates the effect of rough bathymetry on the eddies. If extrapolated globally, the subinertial topographic scales can account for $2.7 \mathrm{TW}$ of work done on the low-frequency circulation, an important sink that is redistributed to mixing in the open ocean.
\end{abstract}

KEYWORDS: Bottom currents; Internal waves; Mixing; Mountain waves; Subgrid-scale processes

\section{Introduction}

Stratified flows passing over bathymetry experience drags that are often many orders of magnitude larger than that effected by skin friction alone, due to the creation of internal motions that either radiate away as internal waves (Bell 1975) or are trapped as "nonpropagating" motions near the topography (e.g., Bacmeister and Pierrehumbert 1988). These motions are important to large-scale momentum, energy, and mixing in both the ocean and the atmosphere, yet often must be parameterized in simulations that do not include enough resolution to capture the small-scale topography.

Here we consider low-frequency flows, where the variation in the flow is taken to be substantially subinertial and has a nearbottom speed $u_{0}$ and stratification $N$. The flow is taken to be over idealized topography that is stochastically specified by empirical models (e.g., Goff and Arbic 2010), where the amplitude is a power-law function of lateral wavenumber $k$ and has a variance

¿ Denotes content that is immediately available upon publication as open access.

Corresponding author: Jody Klymak, jklymak@uvic.ca controlled by a topographic height $h$. In this scenario, the stratified response at a given wavenumber $k$ depends on the ratio of the intrinsic frequency to the Coriolis parameter, $u_{o} k / f$, and the ratio of the obstacle height to depth the flow is able to push the stratified water over the obstacle, $N h / u_{0}$.

For relatively small lateral scale topography, $u_{o} k / f>1$, radiating internal lee waves are possible. If it is also the case that $N h / u_{0} \ll 1$ then linear theory applies, and the drag and energy lost from the mean flow can be calculated analytically (Fig. 1d; Bell 1975). The high-wavenumber part of the topography typically has a small amplitude (so-called "abyssal hills"), so the flows are well approximated by linear theory (Nikurashin and Ferrari 2010; Nikurashin et al. 2014). Estimates for energy put into these motions has ranged from 0.2 to $0.5 \mathrm{TW}(1 \mathrm{TW}=$ $10^{12} \mathrm{~W}$; Naveira Garabato et al. 2013). In addition to exerting drag, a portion of the energy in the radiated waves can be lost via wave-wave interactions to generate a halo of turbulence up to a few hundred meters above the sea floor. Recent work has indicated that parameterizing the mixing and drag due to tidal flow (e.g., Melet et al. 2013; de Lavergne et al. 2017) and mean flows (Melet et al. 2014) over abyssal hills can alter the largescale circulation and stratification. For the case where $u_{o} k / f>1$, but $N h / u_{0}$ is not small, it is believed that partial blocking can enhance the drag and energy loss further, with estimates of up to 


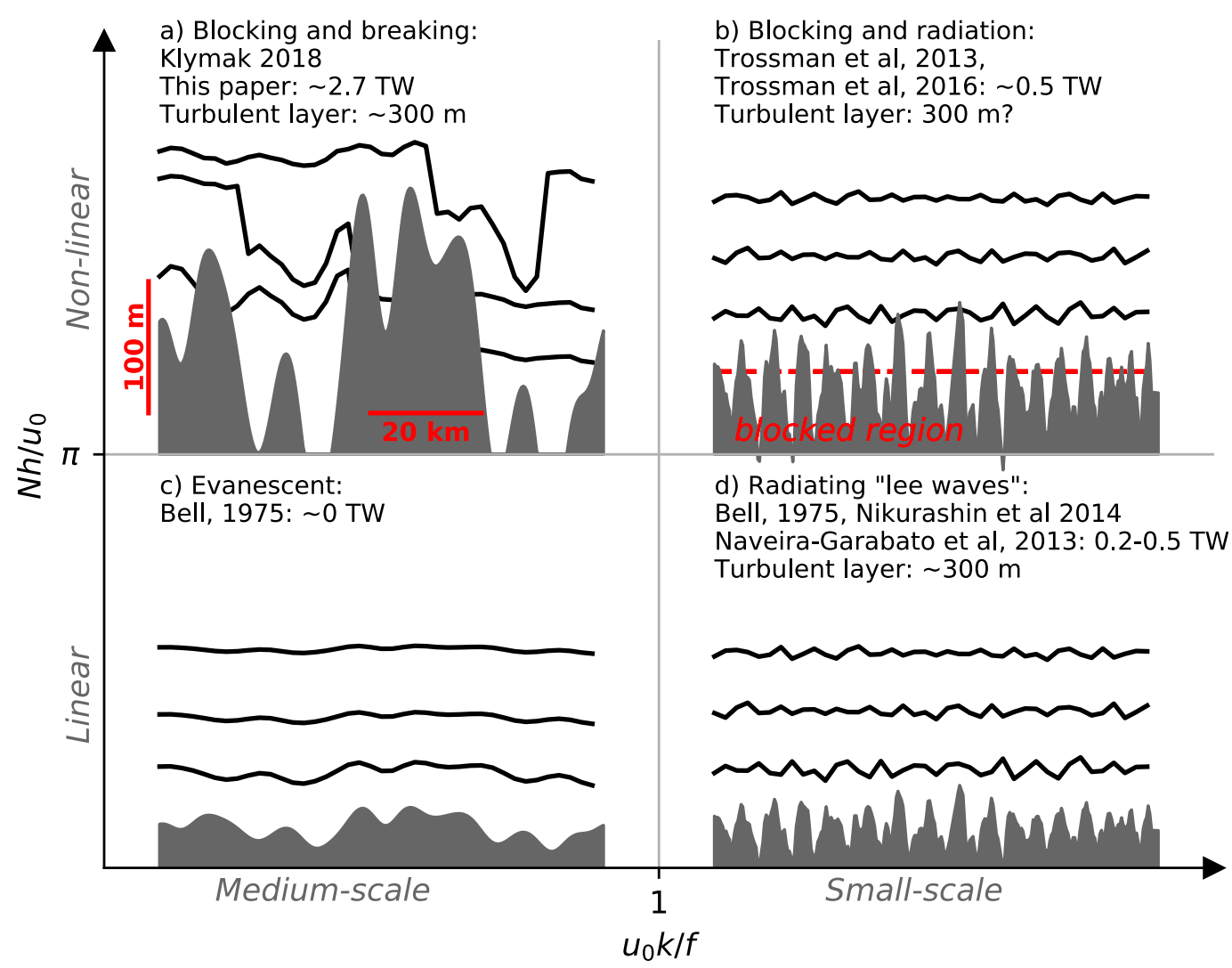

FIG. 1. (a)-(d) Regime diagram for low-frequency stratified $(N)$ flows with speed $u_{0}$ over topography with height $h$, and dominant horizontal wavenumber $k$. The topography in (a) and (d) are filtered from the same topographic spectrum and are plotted with the same relative amplitudes. Panel (c) is a reduced version of (a), and (b) is an exaggerated version of (d). Global estimates of work done against the mean flow, and example papers that have covered these are shown in each region of the diagram.

another 0.5 TW of energy lost due to such motions (Trossman et al. 2013; Fig. 1b).

For medium-scale topography $\left(u_{o} k / f>1\right.$, but still subgrid scale to a climate model) radiating internal waves are not possible, and, in the steady state of the linear inviscid limit, cannot remove energy from the mean flow (Fig. 1c). However, the height scale of the topography $h$, appropriate at medium scale, tends to be substantially larger than for small-scale topography, and $N h / u_{0}$ can become quite large (Fig. 1a). The flow at these scales is inherently nonlinear, and there is substantial local blocking upstream of topographic features, and breaking downstream. Klymak (2018) demonstrates that, for a typical bathymetric spectrum (e.g., the ones used by Nikurashin et al. 2014), the large-scale bathymetry was responsible for $66 \%$ of the total dissipation in the flow, and is crucial to include either via explicitly resolving the flow or by parameterization. This is called nonpropagating drag, because even though the response has drag, it is not classical linear wave drag.

The importance of parameterizing drag and turbulence due to nonpropagating motions over and around large-amplitude topography $\left(N h / u_{0} \gg 1\right)$ has been long realized as necessary to simulate the large-scale atmosphere (e.g., Bacmeister and Pierrehumbert 1988; Lott and Miller 1997). A number of schemes have been devised based on the fact that substantial portions of the flow can be blocked and diverted around an isolated obstacle. The drag typically used is the same as for a bluff body in unstratified flow, is quadratic with the flow speed, and is proportional to the cross-sectional area of the obstacle exposed to the mean flow, with an empirically derived drag coefficient (e.g., Scinocca and McFarlane 2000; Garner 2005). These bluff body parameterizations of the nonpropagating drag have been shown to be important in numerical simulations of the ocean (Trossman et al. 2013, 2016), and to have promise when compared to observations (Trossman et al. 2015), though the ocean work has been in the regime where $u_{o} k / f>1$. These parameterizations are quadratic in flow speed and linear in obstacle height corrected by the blocking depth $h_{b}=u_{0} / N$, i.e.,

$$
D_{\mathrm{np}} \sim\left(h-h_{b}\right) u_{0}\left|u_{0}\right| .
$$

Conversely, Klymak et al. (2010) found that drag over an isolated two-dimensional obstacle for $N h / u_{0} \gg 1$ is linear in $u_{0}$ and quadratic in height:

$$
\frac{D_{\mathrm{np}}}{\rho_{0}} \approx \frac{u_{0}^{2} h}{L} \frac{\pi}{2}\left[\frac{N h}{u_{0}}+\pi\right]=C_{l} u_{0}+C_{q} u_{0}^{2},
$$


where $L$ is an along-flow lateral scale (discussed below), and we have defined a linear drag coefficient as $C_{1}=N h^{2} \pi /(2 L)$ and a quadratic drag coefficient as $C_{q}=h \pi^{2} /(2 L)$. Note that the quadratic (in $u_{0}$ ) term has the same scaling as the nonlinear drag typically used in the atmosphere for low $N h / u_{0}$, but asymptotes to a linear drag law at high $N h / u_{0}$. The physical difference is that the drag for a stratified flow over an obstacle arises due to blocking of the densest part of the flow, creating a cross-obstacle pressure gradient that is solely due to the increased amount of dense water upstream compared to downstream, whereas drag in the bluff body is due to lateral flow separation.

The goal of this paper is to argue that this "medium-scale" topography is important for the ocean, and to test the appropriateness of the parameterization in Eq. (2), and argue that it provides an accurate yet simple way to transition between highand low- $N h / u_{0}$ flow regimes with a minimum of fit parameters. It was shown in Klymak (2018) that the nonpropagating drag is indeed linear in the flow speed $u_{0}$, and not quadratic. Here we test the rest of the parameterization via idealized simulations that vary $N, h$, and the Coriolis parameter $f$, and via more realistic, but still idealized, wind- and thermally forced channel flows. After specifying the numerics used in the simulations (section 2), we argue that Eq. (2) is indeed appropriate across a large range of parameters (section 3 ). The paper subsequently considers a number of quasi-realistic simulations that are idealizations of the flow in the Southern Ocean (section 4). The wind and thermally driven channel was chosen for testing the parameterization because it is a closed system that still demonstrates substantial complexity that may be met by an eddy-resolving, large-scale simulation, and there is substantial literature that uses it already (e.g., Abernathey and Cessi 2014; Marshall et al. 2017). In the discussion (section 5) we outline some caveats with our approach, and then apply the parameterization to a global dataset of topographic roughness, bottom stratification, and velocity from an eddy-permitting assimilated model, to arrive a total energy removed from low-frequency motions of $2.7 \mathrm{TW}$.

\section{Model configurations}

Two types of simulation are used in this paper. The first is a doubly periodic domain forced with a cross-channel body force that, absent other forces, is geostrophically balanced by an along-channel flow. Stratification, topographic roughness, and Coriolis frequency are varied in this setup to test the parameterization of the nearbottom drag. The second configuration is a long reentrant channel that has a large Gaussian ridge in the middle and varying roughness. This model is forced with a wind stress and thermal relaxation at the surface, and the bottom drag is either resolved or parameterized.

The MITgcm was used for all simulations (Marshall et al. 1997), in a manner analogous to previous work at similar scales (Buijsman et al. 2014; Klymak et al. 2016; Klymak 2018). Background explicit vertical and horizontal viscosity and diffusivity are kept low $\left(K_{\rho}=\nu=10^{-5} \mathrm{~m}^{2} \mathrm{~s}^{-1}\right)$ except in the presence of resolved density overturns, where the vertical viscosity and diffusions are increased in a manner consistent with the expected Thorpe scale (Klymak and Legg 2010). There is also numerical diffusivity and dissipation due to the second-order flux-limiting temperature advection scheme (tempAdvScheme $=77$; see the MITgcm manual). Using a weak explicit diffusivity allows the internal wave field to evolve freely to the extent that the chosen resolution allows, rather than adding artificial damping (Shakespeare and Hogg 2017). For the work carried out here, the terms in the energy budget are all calculated and the residual is identified as the dissipation. However, the spatial distribution of explicit dissipation (calculated from the explicit viscosities and local shears) is similar to the spatial distribution of the residual of the energy budget. The model is run in hydrostatic mode for all simulations. Stratification is via a linear equation of state, with temperature as the only active tracer.

\section{a. Doubly periodic simulations with body force}

These simulations are identical to those used in Klymak (2018), and inspired by Nikurashin et al. (2014). We assume a doubly periodic domain with constant stratification and a mean flow in the $x$ direction forced over rough topography. The strength of the flow maintained by a body force meant to simulate an externally imposed surface pressure gradient. The doubly periodic lateral domain of $409.6 \mathrm{~km}$ in the $x$ direction and $118.4 \mathrm{~km}$ in the $y$ direction is chosen to capture sufficient variance in the large-scale topography. All simulations had a constant vertical resolution of $10 \mathrm{~m}$ over $4000-\mathrm{m}$ depth; $1-\mathrm{km}$-scale runs were spun up for $200 \mathrm{~h}$.

The topography is the low-passed version of that used in Nikurashin et al. (2014) and (Klymak 2018) based on Goff and Arbic (2010), with parameters given by

$$
P_{2 D}(k, l)=\frac{2 \pi a^{2}(\mu-2)}{k_{0} l_{0}}\left(1+\frac{k^{2}}{k_{0}^{2}}+\frac{l^{2}}{l_{0}^{2}}\right)^{-\mu / 2},
$$

where $a$ is the root-mean-square of the topographic amplitude, $\mu=3.5$ is a parameter setting the high-wavenumber slope of -1.75 , and $k_{0}=l_{0}=1.84 \times 10^{-4} \mathrm{rad} \mathrm{m}^{-1}$ are parameters that set the wavelength at which the spectrum of the topography starts to flatten out toward a white low-wavenumber spectrum. A range of values were tested for $a$, the background stratification $N, k_{0}$, and $f$. Note that the $h$ used in Eq. (2) is a trough-to-peak height, and hence is approximately $h=2 a$.

\section{b. Wind-forced channel simulations}

The second set of simulations are run in a zonal channel forced by a sinusoidal wind stress and temperature relaxation at the surface. The wind-forced domain has the same numerics as the simulations above. The channel is $1024 \mathrm{~km}$ wide and $1600 \mathrm{~km}$ long (Fig. 2). The bathymetry is $3000 \mathrm{~m}$ deep, with sloping walls along the north and south wall faces, and a meridional Gaussian ridge in the middle of the domain with a zonal lateral scale of $100 \mathrm{~km}$ and a height of $1000 \mathrm{~m}$ (2000-m depth). Simulations are run with smooth seafloor bathymetries, and bathymetries with an added roughness that follows the same statistics as in the previous section (Fig. 3). These runs are started with a uniform temperature of $4^{\circ} \mathrm{C}$, and the temperature is forced at the surface with a relaxation time scale of $48 \mathrm{~h}$ to be $4^{\circ} \mathrm{C}$ at the southern end, and linearly increasing to $12^{\circ} \mathrm{C}$ at the northern end. A sinusoidally varying westerly wind forcing is also applied down-channel, with a peak in the center of the channel of $0.2 \mathrm{~N} \mathrm{~m}^{-2}$, and nulls at the north and south walls. This forcing and setup are meant to emulate many wind-driven channel studies (e.g., Abernathey and Cessi 2014; Marshall et al. 2017). 

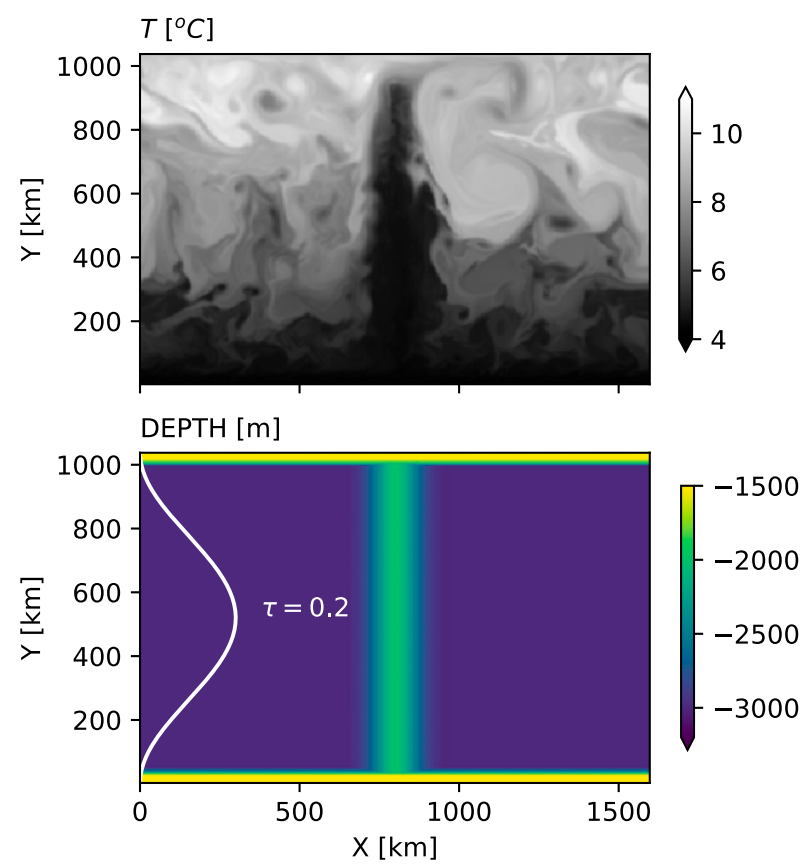

FIG. 2. Wind-forced channel configuration: (top) surface temperature from the base simulation and (bottom) the smooth bathymetry domain with a large-scale ridge in the middle. The wind profile is shown in the lower panel, with a peak $\tau=0.2 \mathrm{~N} \mathrm{~m}^{-2}$ at the center of the channel.

The simulations were run in stages; a $20-\mathrm{km}$ horizontal resolution and $80-\mathrm{m}$ vertical resolution simulation was run for 100 years with the smooth bathymetry. A second run was conducted with the lateral resolution increased to $5 \mathrm{~km}$, and the vertical resolution increased to $40 \mathrm{~m}$, for 10 years. $5 \mathrm{~km}$ was the limit of what we could feasibly simulate at a manageable computing cost, and the indication from Klymak (2018) is that this resolution will provide a drag in flows with rough bathymetry that is probably exaggerated by a factor of 1.4 compared to simulations with respective horizontal and vertical resolutions of 100 and $10 \mathrm{~m}$. As noted below, the simulations are close to being in mechanical equilibrium, but they are not anywhere near thermal equilibrium, which would require several centuries of simulation [e.g., Munday et al. (2015) ran similar simulations for 620 years].

\section{Parameterizing drag due to "medium" horizontal-scale topography}

The first series of simulations were run with a body force over doubly periodic stochastic topography similar to Klymak (2018). Here we only consider topography low-passed in space so that variance at horizontal scales smaller than $6 \mathrm{~km}$ is attenuated. At smaller scales, linear lee-wave parameterizations tend to do well, so we are only interested in parameterizing the large scales here. For comparison with the parameterization [Eq. (2)], we choose a representative horizontal spacing between obstacles of $L=(100 \mathrm{~km}) 1.8 \times 10^{-4} \mathrm{rad} \mathrm{m}^{-1} / k_{0}$, where $k_{0}$ is the roll-off wavenumber of the topographic spectrum (Klymak 2018). Simulations were run with a base

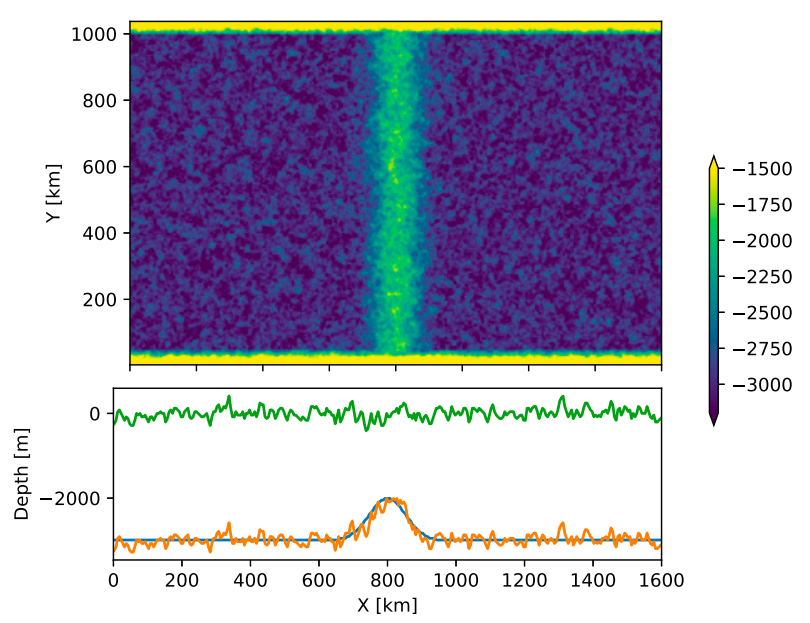

FIG. 3. Wind-forced channel bathymetry, with stochastic roughness added: (top) a plan view and (bottom) a representative cross section. The stochastic part of the bathymetry is shown in green.

configuration of $f=10^{-4} \mathrm{rad} \mathrm{s}^{-1}, u_{0}=0.1 \mathrm{~m} \mathrm{~s}^{-1}, N=10^{-3}$ rad s ${ }^{-1}$, a maximum depth $H=4000 \mathrm{~m}$, and a topographic roughness on top of this as described by Eq. (3) with a basic amplitude of $a=305 \mathrm{~m}$ and $k_{0}=l_{0}=1.8 \times 10^{-4} \mathrm{rad} \mathrm{m}^{-1}$.

There is important time dependence to these simulations, both due to the initial conditions, and due to mixing in the model domain. The topographic amplitude here is $a=305 \mathrm{~m}$ so the peak-to-trough excursions of the topography here are $h \sim 600 \mathrm{~m}$ (Fig. 4). However, the near-bottom stratification $N(z)$ is eroded with time in these simulations due to mixing, such that the average stratification in the layer encompassing the topography the stratification is half the stratification aloft (Fig. 5). There is no buoyancy source to replenish the stratification, and this stratification is close to steady state by the end of the simulation. This leads to a reduction of drag that we account for by considering the height of the obstacles as WKB-stretched variables for the purposes of the parameterization. So, for the parameterization we use $h_{\mathrm{WKB}}=h\langle N\rangle / N_{0} \approx(1 / 2) h=a$, where $a$ is the topographic amplitude. Note that in Klymak (2018) we simply used $a$ for the parameterization without justification. Conversely, we note below that the freely evolving wind-driven simulation that includes an overturning circulation with a buoyancy source feels the full crestto-trough bathymetry, and a WKB adjustment is not necessary.

Energy budgets were performed on the runs, as described in Klymak (2018), and an average vertically integrated dissipation rate $D\left(\mathrm{~mW} \mathrm{~m}^{-2}\right)$ across the domain was computed as the integral of $v F_{b}$, where $F_{b}$ is the $y$-direction body force used to force the model, and $v$ the north-south velocity (Fig. 5a,b).

Simulations were carried out as deviations from the base values. First, $a$ was varied, with $a=155,305$, and $610 \mathrm{~m}$ (Fig. 6a); we find that higher obstacles lead to more dissipation. The scaling is not linear in $a$, but rather closer to quadratic. We are prevented from exploring smaller-scale topography by the vertical resolution of the model setup.

As the length scale $L$ is changed by varying $k_{0}$ (Fig. 6b), we see that there is less dissipation with increasing length scale as expected. The dissipation appears to plateau for small horizontal 

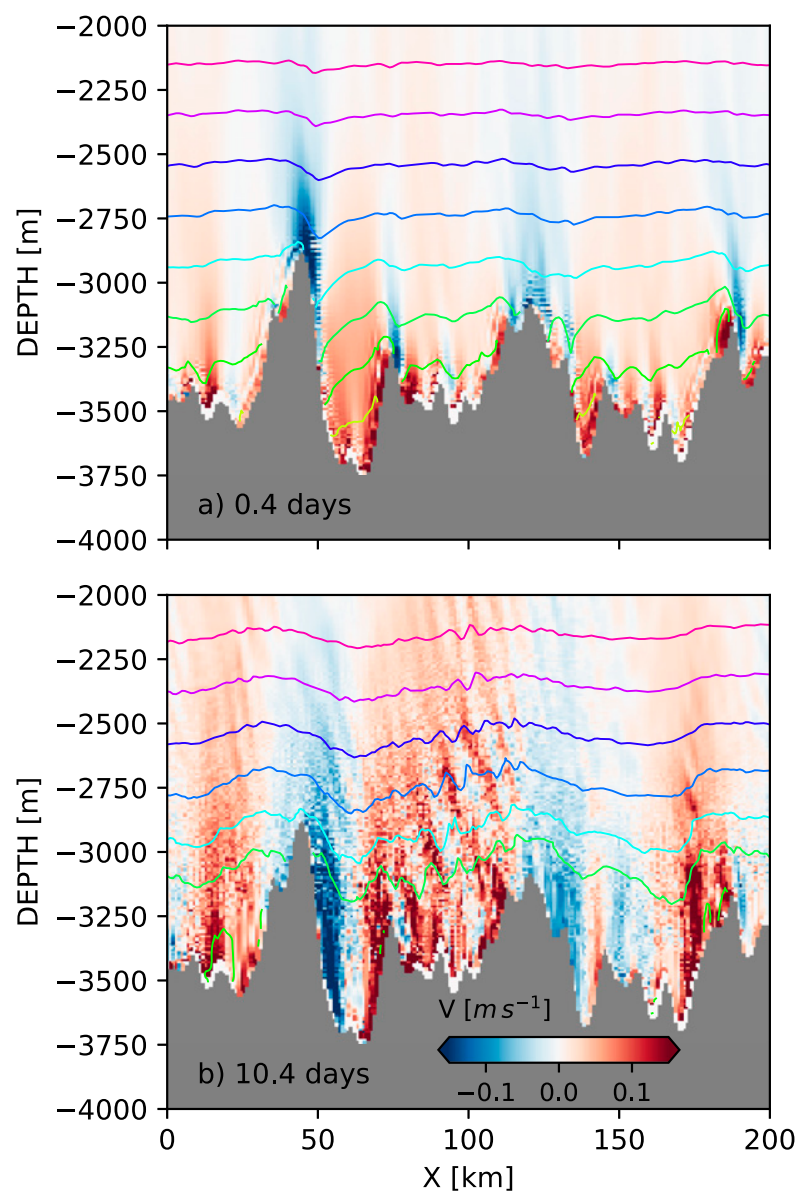

FIG. 4. Flow snapshots over a slice of the topography for the base body-forced simulations $\left(f=10^{-4} \mathrm{rad} \mathrm{s}^{-1}, N=10^{-3} \mathrm{rad} \mathrm{s}^{-1}, a=\right.$ $305 \mathrm{~m}$, and $U_{0}=0.1 \mathrm{~m} \mathrm{~s}^{-1}$ at (a) 0.4 days and (b) 10.4 days. Crosschannel flow $V$ is shown as colors and the isopycnals are contoured every $\sigma_{\theta}=0.2 \mathrm{~kg} \mathrm{~m}^{-3}$ in each panel.

scales, mildly disagreeing with the scaling here. This is still under investigation, but is probably because the topographic elements become less dynamically distinct.

As stratification is increased, $N=0.5,2,4 \times 10^{-3} \mathrm{rad} \mathrm{s}^{-1}$ (Fig. 6c), we see that dissipation goes up, as predicted. This is the starkest difference with the nonpropagating drag parameterizations used in previous literature (e.g., Trossman et al. 2013), which do not account for stratification except as a second-order correction. These simulations show that for larger values of $N h / u_{0}$, the stratification plays a leading-order role.

There appears to be, at most, a weak dependence on the Coriolis force (Fig. 6d), again as predicted by Eq. (2) where there is no dependence on the Coriolis parameter. The agreement is not perfect; there is a $30 \%$ drop in dissipation as the Coriolis parameter drops to the value that corresponds to $15^{\circ} \mathrm{N}$. However, here we have again not changed the topography, so as $f$ drops more internal waves are able to radiate rather than being blocked, a complication we have not tried to account for.

Finally, Klymak (2018) showed a clear dependence on $u_{o}$ that follows that predicted by Eq. (2), so we do not reproduce that here. a) $\mathrm{D}\left[\mathrm{mW} \mathrm{m}^{-2}\right]$

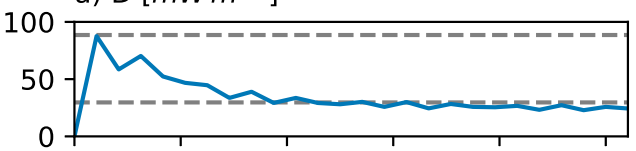

b) $10^{-7} \mathrm{D}\left[\mathrm{m}^{2} \mathrm{~s}^{-3}\right]$

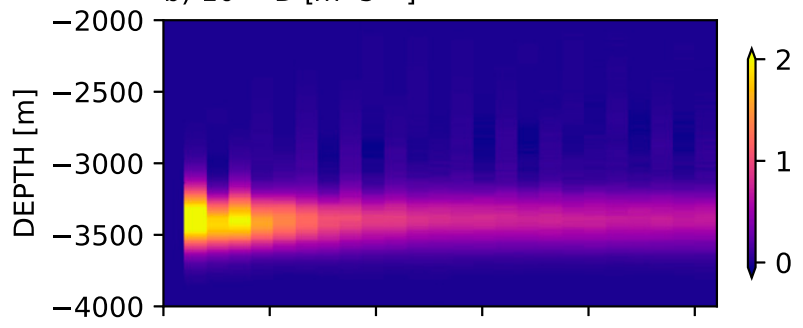

c) $10^{-3} \mathrm{~N}\left[\mathrm{~s}^{-1}\right]: 3200-3800 \mathrm{~m}$

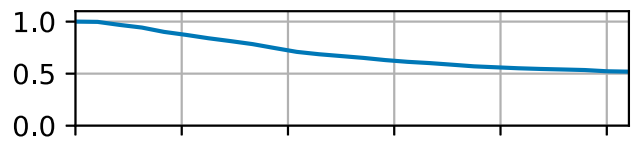

d) $10^{-3} \mathrm{~N}\left[\mathrm{~s}^{-1}\right]$

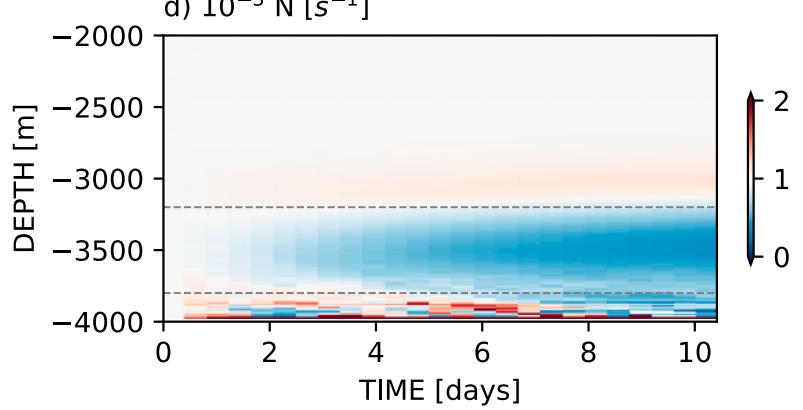

FIG. 5. Time series of (a) total average dissipation in the base bodyforced simulation, as calculated from the work being done by the external forcing. The upper dashed line is the parameterization proposed here with the peak-to-trough topographic height $h=2 a$. The lower dashed line is for $h=h_{\mathrm{WKB}} \approx a$, accounting for the loss of stratification in the bottom layer. (b) Depth where the largest energy is input into the model (averaged laterally across the domain). (c) Square root of average stratification $N^{2}$ between 3200 and $3800 \mathrm{~m}$. (d) Square root of the lateral average of stratification in the bottom $2000 \mathrm{~m}$; gray dashed lines indicate the depth integrated over for (c).

Overall, the parameterization appears to do well over a wide range of ocean-relevant conditions. It has the advantages over other schemes of its containing only one ad hoc parameter, $L$ in Eq. (2), and its allowance of a seamless merging of the parameterizations for flows with high and low $N h / u_{0}$ without arbitrary cutoff parameters. Conversely, the parameterization does not include the high-wavenumber component of the topography, and hence misses the internal wave dissipation, but there have been robust attempts at addressing this portion of the drag problem already (Nikurashin et al. 2014).

\section{Wind-forced channel parameterization}

The doubly periodic simulations forced via a body force are not very applicable to the open ocean, where mean flows tend 
a) Topographic amplitude

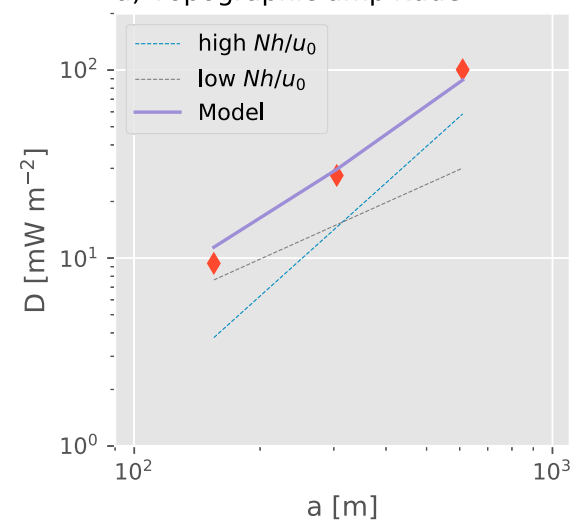

c) Stratification

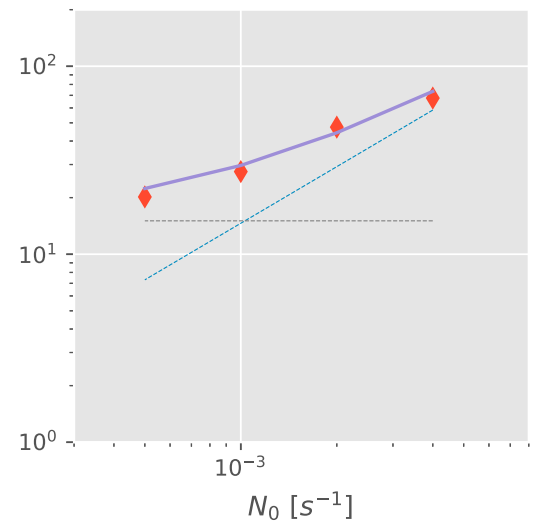

b) Rolloff wavelength

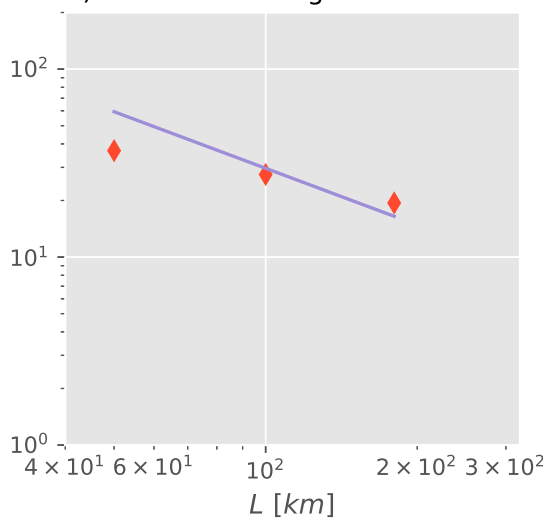

d) Coriolis

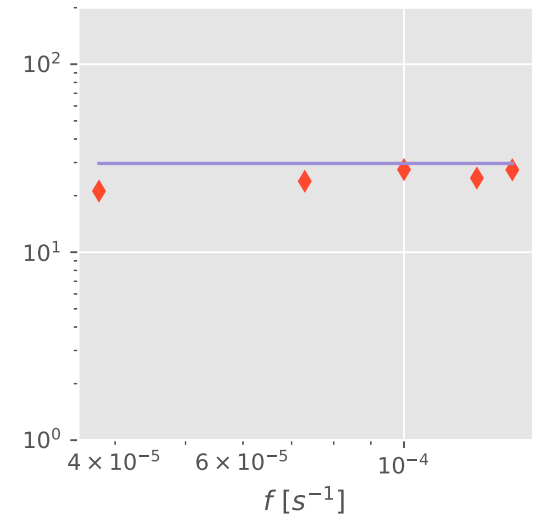

FIG. 6. Integrated dissipation $D$, in simulations that vary: (a) stochastic topographic amplitude, (b) the roll-off wavelength at which the topographic amplitude starts to fall off, (c) the stratification, and (d) the Coriolis parameter. In each panel, the expectation from the model given by Eq. (2) is indicated, with asymptotes for both the high and low $N h / U$ shown as thin dashed lines.

to be dominated by eddying motions with subinertial time scales. Here the parameterization is tested in a wind- and thermally driven simulation that is forced only at the surface, allowing the near-bottom drag to evolve on its own, and buoyancy to be replenished by the overturning circulation in the channel. The simulation chosen was a wind-forced reentrant channel with relaxation of the surface temperature to a north-south gradient, and a large-scale Gaussian ridge in the middle of the channel. This style of simulation catalyzes eddies in the flow (Abernathey and Cessi 2014), and eddy strength, and hence downstream transport and stratification, have been shown to be sensitive to the parameterization of the bottom drag (Marshall et al. 2017).

Smooth-bathymetry simulations were run with either a weak quadratic bottom drag $\left(C_{q}=10^{-3}\right)$ or with a bottom drag based on the parameterization above [Eq. (2)], meant to simulate the drag effect of the rough bathymetry. This was implemented as a quadratic plus linear drag in the model, using an approximate near-bottom stratification of $N \approx 5 \times 10^{-4}$ $\mathrm{rad} \mathrm{s}^{-1}$, a topographic amplitude of $a_{0}=305 \mathrm{~m}$, and a lateral scale of $L=100 \mathrm{~km}$. Using $h=2 a_{0}$ to account for the peak-totrough amplitude, this yields a base run linear coefficient of $C_{l}=2.9 \times 10^{-3} \mathrm{~m} \mathrm{~s}^{-1}$ and a quadratic coefficient of $C_{q}=3 \times 10^{-2}$.
A better parameterization would be to make the coefficients depend on the evolving near-bottom stratification, rather than choosing a value for $N$; however, that complexity was not addressed in these simulations.

Finally, drags are usually applied at the bottom-most cell of the model. However, this leads to excessive near-bottom shear, and does not slow the water down over the range of depths that it does in the simulations with rough bathymetry. To simulate this effect, the bottom drag is evenly distributed over a vertical scale of $280 \mathrm{~m}$ ( 7 grid cells) similar to the vertical blocking scale of the topography $\pi u_{0} / N$ (Klymak et al. 2010). This is analogous to what was done by Trossman et al. (2016), who applied their nonpropagating drag over the bottom $500 \mathrm{~m}$.

\section{a. Flow response}

Example lateral slices of temperature through the simulations show similar features between the Smooth and Rough simulations (Fig. 7), but small scales are suppressed in the Rough simulation leading to a more ordered eddy field near the surface as the cascade to small scales is hampered. The Param simulation has similarly suppressed eddy filamentation. Note that all three simulations have different mean temperatures at 


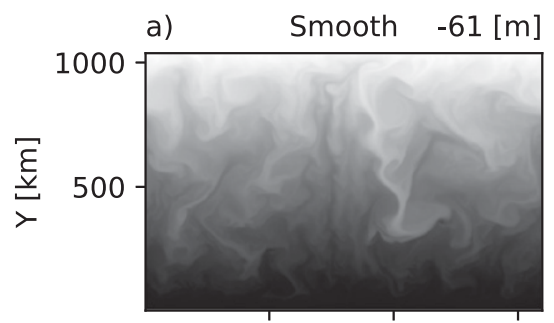

b)

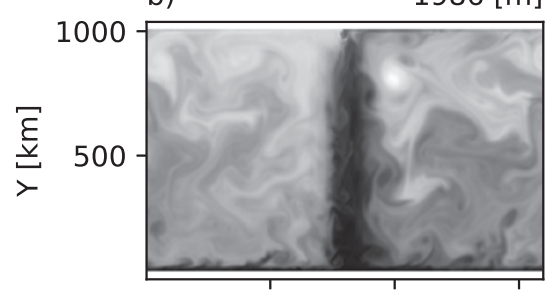

c)

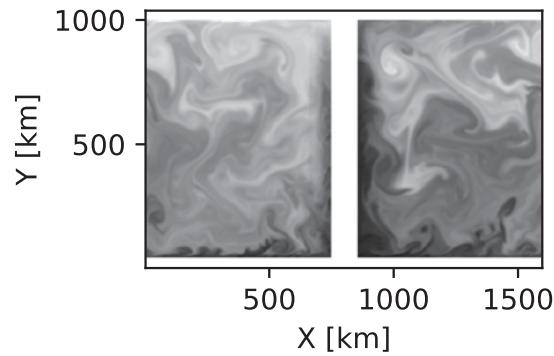

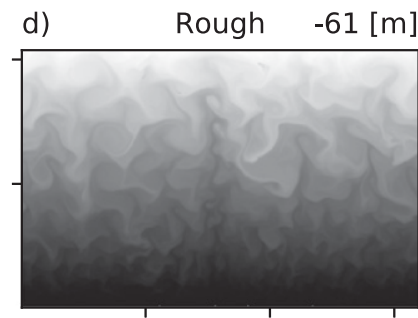

e)

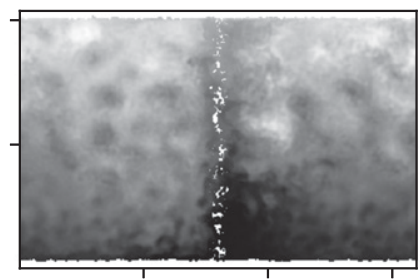

f)

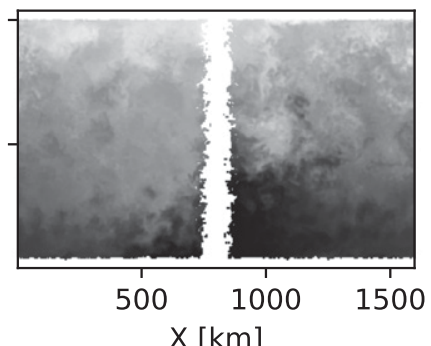

g) Param -61 [m]

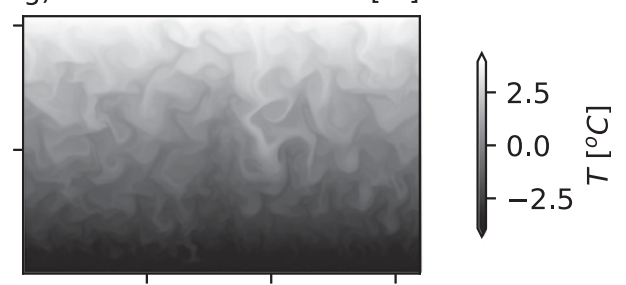

h)

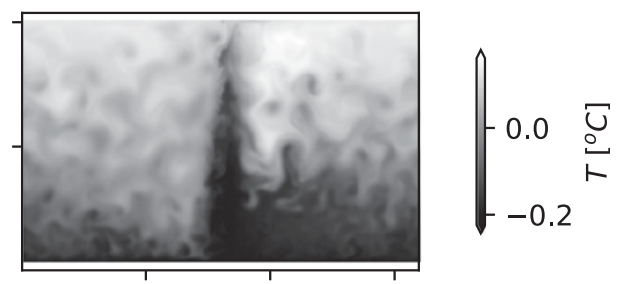

i)

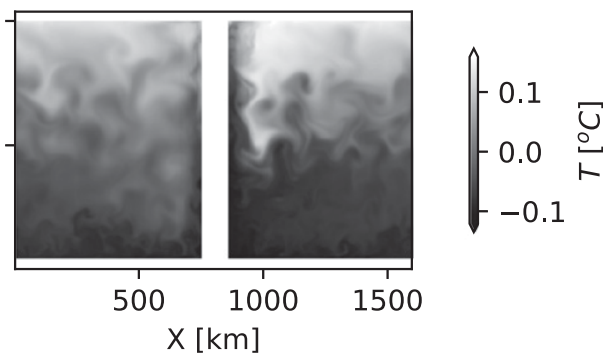

FIG. 7. Example lateral temperature slices (as anomalies from the mean at that depth) through the simulations at depths of $-61,-1986$, and $-2477 \mathrm{~m}$, with (a)-(c) smooth bathymetry, (d)-(f) a synthetic rough bathymetry, and (g)-(i) an enhanced parameterized drag.

these depths, a symptom of the fact that none of the runs are in thermal equilibrium.

Spectra of vertical velocity (Fig. 8) clearly show the suppression of eddies at subinertial frequencies in the Rough and Param simulations, where there is less variance following the expected eddy propagation curve. Note the relatively weak internal wave field in all three simulations, which is unsurprising because any trapped internal waves would be subinertial in the Eulerian frame of reference, and the usual sources of propagating internal wave energy are missing (e.g., tides and variable winds).

The effect on the large-scale flow is as predicted by other studies (e.g., Marshall et al. 2017). Suppressing the mesoscale eddy field increases the baroclinicity of the flow significantly, as well as deepening the thermocline (Fig. 9). The reason for this is that eddies are necessary to transmit surface stress down through the water column to ultimately be removed at the bottom. If the eddies are spun down more quickly, the flow must develop more baroclinicity, and hence more baroclinic instabilities, until a sufficiently large eddy stress exists to allow the flow to come into steady state. A by-product is that there will be a stronger near-surface flow, and an increase in the transport along the channel (see below).

An interesting effect of the rough bathymetry (and its parameterization) is to greatly alter the standing meander that forms directly downstream of the basin-scale ridge, most clearly seen by looking at the bottom pressure signal (Fig. 10).
There is low pressure over the ridge in all three simulations, but downstream the large anticyclone is not as strong in the Rough and Param simulations. The effect of this on the large-scale drag is beyond our scope here, but is a curious result of adding more friction to the bottom drag (e.g., Thompson and Naveira Garabato 2014).

\section{b. Integrated response and parameterization}

The overall effect of the rough topography and its parameterization is to lead to smaller eddy kinetic energy by approximately $50 \%$ (Fig. 11a), and stronger downstream transport by up to a factor of 4 (Fig. 11c). The time series also make it clear that the simulations are in approximate mechanical steady state (Fig. 11a), but that they have not reached thermal equilibrium (Fig. 11b), which would take a few hundred years of simulation.

Parameterizing the drag due to the rough bathymetry was attempted in a few different ways. First, we tried a run with just a quadratic drag coefficient of $C_{Q}=0.05$, and a separate run with just a linear drag coefficient of $C_{L}=0.0025$. These are quite high drag coefficients and lead to a strong increase of the down-channel transport (Fig. 11c), to 35 and $30 \mathrm{~Sv}$ ( $1 \mathrm{~Sv} \equiv$ $\left.10^{6} \mathrm{~m}^{3} \mathrm{~s}^{-1}\right)$, respectively. Applying the parameterization with $h=2 a=610 \mathrm{~m}$ leads to a much closer fit to the downstream transport (Param, Fig. 11c, red curve).

In these simulations, it is easiest to change the topographic amplitude, so we have done so over a range of topographic 
a) Smooth

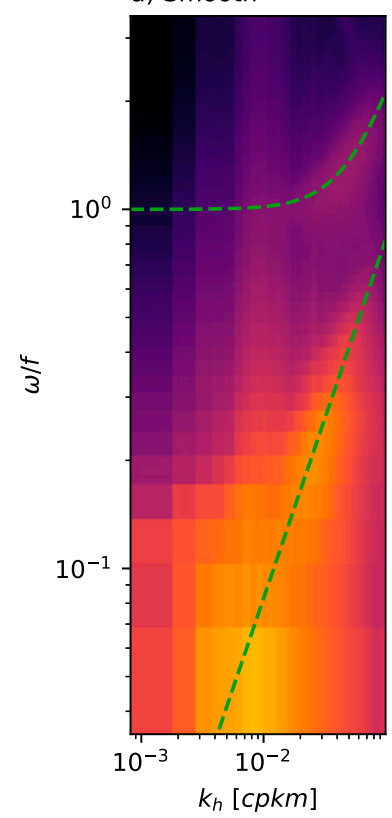

b) Rough

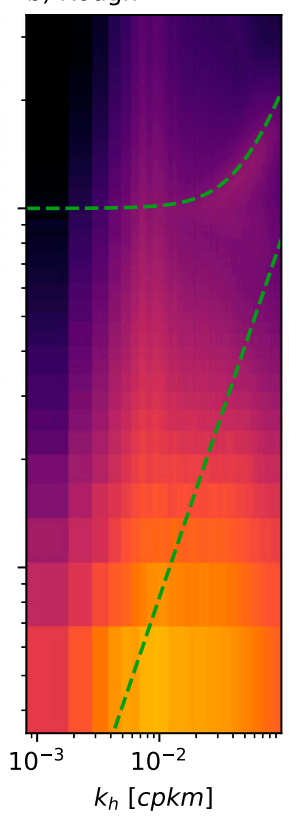

c) Param

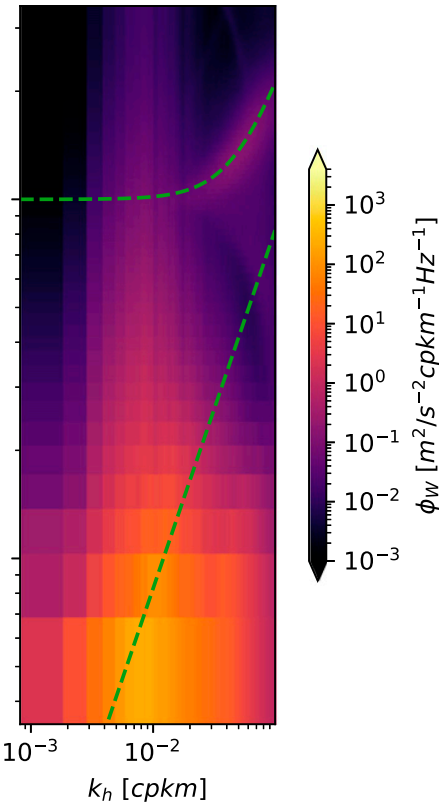

FIG. 8. Two-dimensional spectra of vertical kinetic energy at $-1986 \mathrm{~m}$ for the three main simulations. The dashed green lines are a mode- 1 internal wave dispersion relation (at superinertial frequencies) and eddy motion assuming eddy propagation speeds of $8 \mathrm{~km} \mathrm{day}^{-1}$.

heights that are relatively large, $a=305 \mathrm{~m}$ (the cases shown above), $450 \mathrm{~m}$, and $610 \mathrm{~m}$. We ran the model with explicit rough bathymetry, and with the parameterized drags. For the linearonly drag law we increased the coefficient by the square of the topographic height, and for the quadratic-only drag law we increased the coefficient linearly with the topographic height.

As the stochastic topographic amplitude $a$ is increased in the model simulations with explicit roughness, the downstream transport increases from 45 to $62 \mathrm{~Sv}$ (Fig. 12, orange), due to the extra suppression of eddies. The quadratic form drag is assumed to scale with $h=2 a$, and hence does not see as much of an enhancement in downstream transport as $a$ is increased (Fig. 12, gray, dashed). The linear form drag scales as $h^{2}$, and has a larger fractional increase in the downstream transport than is observed (Fig. 12, gray, dash-dot). The parameterization (Fig. 12, red curve) has a dependence on $h=2 a$ that is very similar to the explicit bathymetry simulation. Of course, both the linear or quadratic drags could have been tuned to better match the rough bathymetry simulations for any particular value of $a$, but the point is that the power-law dependence on other values of $a$ would still be incorrect, such that the hybrid scheme is an improvement over both.

\section{Discussion}

Here we tested a hybrid drag parameterization, Eq. (2), and found that it improves the simulation of bottom roughness in coarse models that do not resolve the bathymetry. There are a few straight-forward caveats to this finding that should be noted. First, the body-forced runs (no wind forcing) found that the parameterization should use the amplitude of the topographic spectrum, scaled in a WKB sense to account for a loss of stratification as the simulation evolves: $h_{\mathrm{WKB}} \approx a$ (e.g., Aguilar and Sutherland 2006), in Eq. (3). For the wind-forced runs the near-bottom stratification evolves in response to the buoyancy forcing at the surface, and the flow comes from different directions as eddies move past the topography, therefore well-mixed regions do not form in the deepest topography, and $h=2 a$.

Note that when we applied the drag law to the eddying simulations, we did not include the computational complexity of calculating the stratification, and instead used a constant $N$ for the parameterization. This was just for convenience, and to see if the drag law was useful. However, given that the linear drag depends on $N$, this really should be done online, so that the response to stratification is allowed to vary across the domain. This requires more work in determining $N$ appropriately both in terms of how to average it to apply to this parameterization, and in how to include the effect of mixing due to the rough topography in the evolution of the stratification. Similarly, we would expect substantial mixing from the parameterized drag, but we have not included this extra mixing in the simulations used here; how this would feed back with the overturning circulation in the eddying simulations is an important question (Broadbridge et al. 2016). However, as noted, we did not run the models to thermal equilibrium, so testing this would require significantly more numerical expense.

An important caveat is that all the topography used here is stochastic, isotropic, and homogenous across the domain. These assumptions break down at the larger scales we are interested in, perhaps much more so than the smaller-scale 


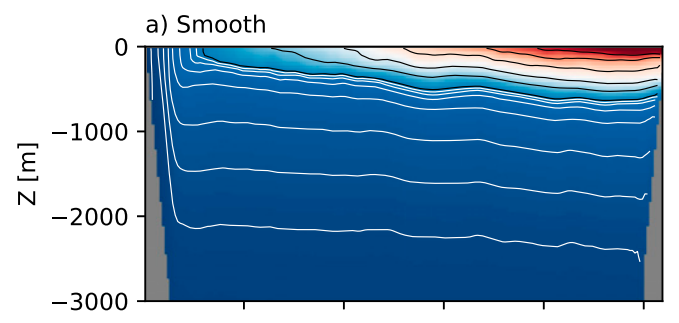

b) Rough

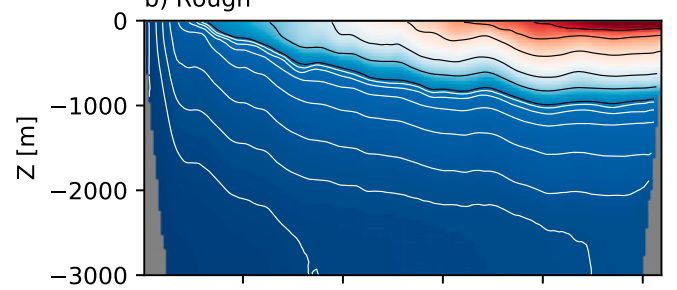

c) Param

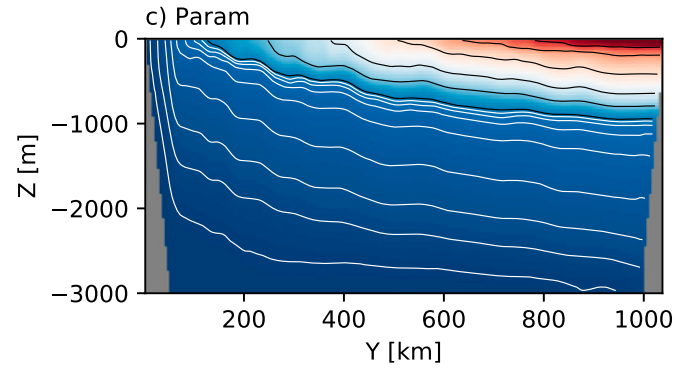

FIG. 9. Zonal temperature sections, averaged from $x=0$ to $500 \mathrm{~km}$. Black contours are every degree Celsius, and white contours are every $1 / 10^{\circ} \mathrm{C}$ from $5^{\circ}$ to $4^{\circ} \mathrm{C}$ for three simulations: (a) smooth bathymetry, (b) rough bathymetry, and (c) smooth bathymetry with the parameterization.

abyssal hills that radiate lee waves. In particular, the effect of organized large-scale bathymetry, as opposed to stochastic bathymetry, should be investigated. Anisotropy may particularly matter if the different topographic wavelengths are organized in such a way as to be random for one direction, and in-phase for others.

Finally, the eddying simulations were somewhat cheaply run, and there is good evidence that changing the horizontal and vertical resolution can affect the near-bottom drag significantly in the presence of rough bathymetry (Klymak 2018). Our coarse $(\sim 40 \mathrm{~m})$ vertical resolution in the wind-forced runs hampered our ability to look at smaller topographic amplitudes.

The relative success of this simple parameterization argues for further consideration of these issues and more study into the underlying physics as it applies to three-dimensional bodies in a rotating fluid. In particular, it is not clear why rotation is so unimportant to the drag. The scaling for drag in high $N h / u_{0}$ flow was for a two-dimensional flow with no rotation following Klymak et al. (2010). Isolated obstacles with rotation exhibit a quadratic drag dependence, whence came the drag laws used in the atmospheric simulations. So, somehow the effects of many, randomly distributed, obstacles combine to give a drag law similar to a two-dimensional obstacle with no rotation. Further simulations are being carried out that attempt to understand this flow regime more completely.

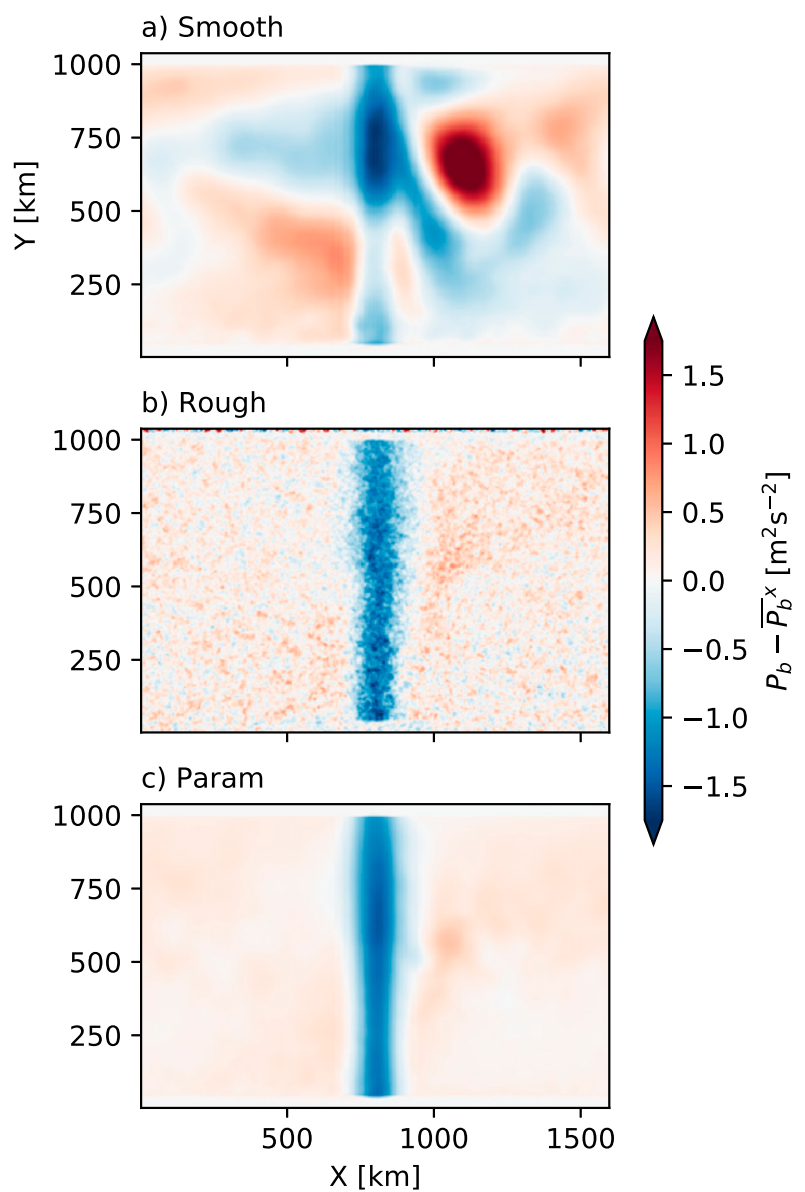

FIG. 10. Bottom pressure averaged over last two years of the simulation with the zonal means removed: (a) smooth simulation with weak drag, (b) rough simulation, and (c) the parameterization of the roughness in a smooth model.

The overall importance of parameterizing the nonpropagating drag with hybrid dependent on $N h / u_{0}$ between a linear drag and a quadratic one can be investigated by calculating the effect on the global ocean. Using the bathymetry roughness parameterization as supplied by Goff and Arbic (2010), the near-bottom stratification from the World Ocean Atlas (Boyer et al. 2013), and the near-bottom velocities from a $1 / 12^{\circ}$ eddy-permitting dataassimilating global HYCOM simulation (Naveira Garabato et al. 2013), drags and energy loss were computed using 5-day averages of bottom velocities from the simulation, and then averaged for the 1-yr simulation to capture any seasonal effects (Fig. 13). The nonpropagating drag can further be compared to the propagating drag due to stationary lee-wave radiation, again from Naveira Garabato et al. (2013).

Using our parameterization, nonpropagating drag from the medium-scale topography $\left(k u_{0} / f<1\right)$ dominates the drag due to lee-wave radiating short-scale topography $\left(k u_{0} / f<1\right)$ almost everywhere in the ocean (Figs. 13a-c,h). Of the quadratic and linear drags proposed here, the linear drag proportion is about $30 \%$ of the total, and hence important, particularly in low-velocity regions (Fig. $13 \mathrm{~g}$ ) where $N h / u_{0}$ is large. 


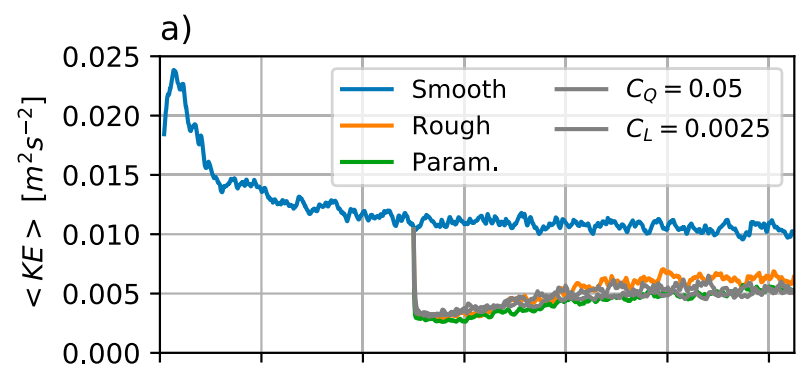

b)

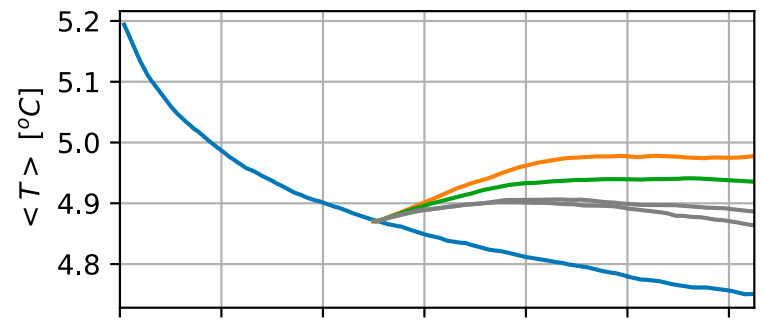

c)

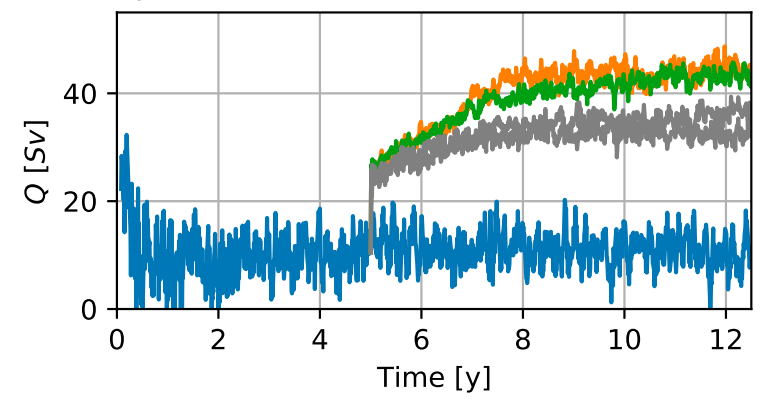

FIG. 11. (a) Model-mean kinetic energy in the simulations with various parameterizations of the drag. (b) Model-mean temperature (c) Mean downstream transport. Smooth is with no roughness and weak quadratic drag, Rough has stochastic bathymetry in the model, and Param is smooth bathymetry with the hybrid linear/quadratic parameterization given in Eq. (2). Here, $C_{Q}$ is with a heightened quadratic drag only, and $C_{L}$ is with a heightened linear drag only.

The medium-scale drag is disproportionately concentrated in the Southern Ocean, with its rough topography and vigorous eddy field.

To our knowledge, simulations have either used the linear or quadratic drag laws exclusively, and not used this hybrid approach. The lee-wave parameterizations (e.g., Nikurashin et al. 2014) work out to be linear drag laws, and the parameterizations that in addition account for nonpropagating effects (Trossman et al. 2013, 2016) also are implemented as a linear drag with a geometric amplification, but just for short-scale topography. Nonpropagating drag in the atmosphere is typically parameterized as a quadratic drag (Lott and Miller 1997; Scinocca and McFarlane 2000). Certainly, there are values of $N h / u_{0}$ that are large enough in the atmosphere to mean that the linear part of the drag law proposed here is likely important.

An obvious caveat of the calculation in Fig. 13 is that the near-bottom velocities in the $1 / 12^{\circ}$ simulation partially depend on the bottom drag used in that simulation, so there is the

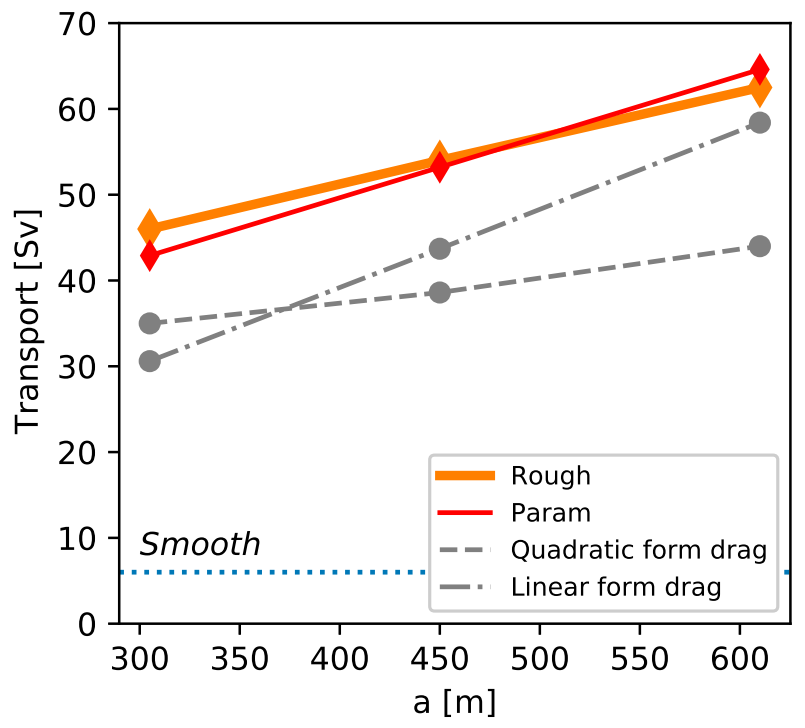

FIG. 12. Effects on the downstream transport of different roughness parameterizations. Rough is the $5-\mathrm{km}$ simulations with resolved topography included. Param (red) is the same parameterization proposed here with $h=2 a$. Simulations with a quadratic (gray dashed) and linear (gray dash-dot) form drag only, approximately scaled to match the Rough simulations.

potential that new global simulations that directly include the drag law proposed here would yield different total work. However, the simulation we used did have data assimilation, and hence was constantly being nudged to be more realistic, so we feel the near-bottom velocities here are a reasonable first estimate.

Whether a drag law and accompanying mixing as proposed here needs to be implemented in higher resolution numerical simulations depends on the resolution and the bathymetry. A high-resolution eddy-permitting or eddy-resolving model may have grid resolutions on the order of $10 \mathrm{~km}$, which after lateral diffusivities are accounted for, lead to resolving flow features on the order of 30-50 km, and topography on the scale of 20$30 \mathrm{~km}$. This would fall right in the middle of the topographic wavelength range from 180 to $6 \mathrm{~km}$ we are considering here, and hence a substantial fraction of the drag and work discussed here may actually be resolved. As pointed out in Klymak (2018), it is further important to have enough vertical resolution to simulate these drags, and even eddy-resolving simulations may have poor near-bottom resolution. Conversely, once we get to large-scale model resolutions that do not resolve the topography discussed here, it is likely they do not have the near-bottom eddying motions that create the large nearbottom velocities, and they deal with lateral diffusivities using schemes based on Gent and McWilliams (1990). So, while our results indicate that this is an important part of the flow regime, how much of this drag is captured in a given simulation depends crucially on its resolution (both vertical and horizontal) and the scale at which the bathymetry is provided. Given that modern bathymetry has approximately 9-km resolution (Tozer et al. 2019) with robust bathymetric spectra, it is possible that many finescale realistic simulations will not 

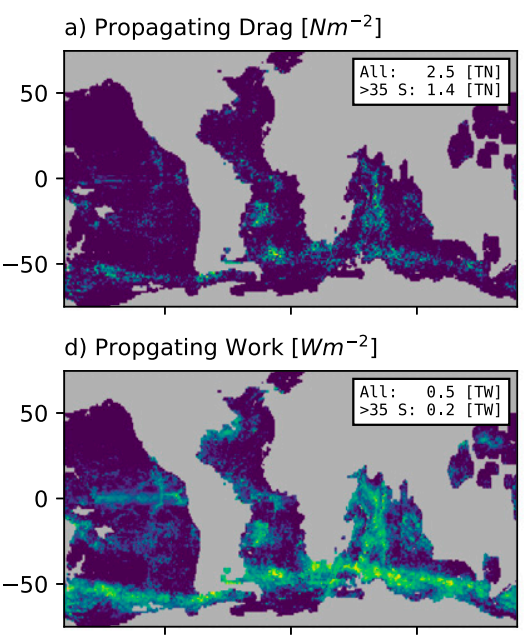

g) Linear/(Linear+Quadratic Drag)

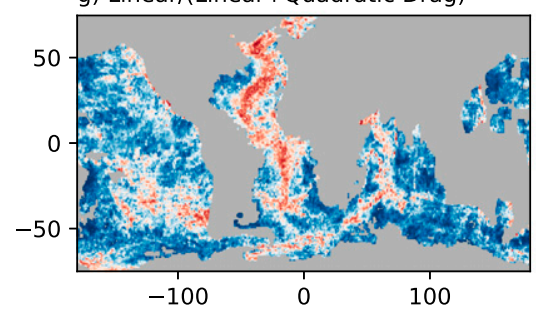

b) Quadratic Drag [ $\left.\mathrm{Nm}^{-2}\right]$

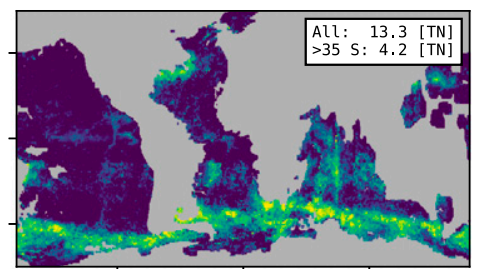

e) Quadratic Work $\left[\mathrm{Wm}^{-2}\right]$

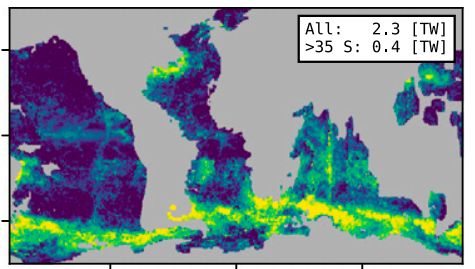

h) Non-propagating Drag/ All Drag

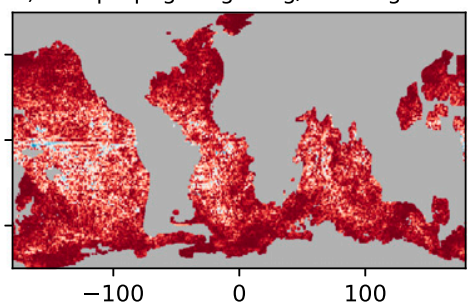

c) Linear Drag $\left[\mathrm{Nm}^{-2}\right]$

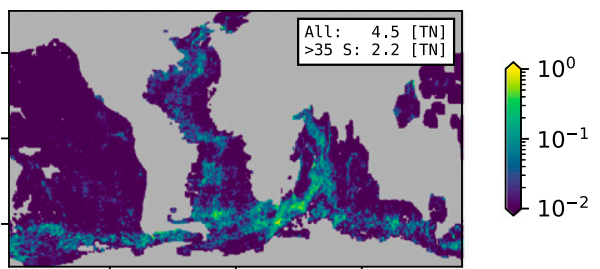

f) Linear Work $\left[\mathrm{Wm}^{-2}\right]$

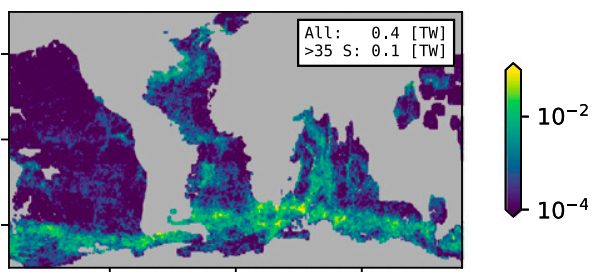

i) Non-propagating Work/ All Work

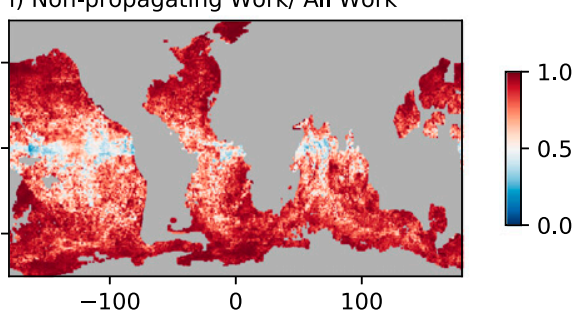

FIG. 13. Bottom drag, work done on the low-frequency flow, and ratios. Near-bottom velocities are from the $1 / 12^{\circ} \mathrm{HYCOM}$ model run described in Naveira Garabato et al. (2013), stratification is taken from the World Ocean Atlas 2013 (Boyer et al. 2013), and bottom topography parameters are taken from Goff and Arbic (2010). (a) Propagating drag (radiating lee waves), as described in Naveira Garabato et al. (2013). (b) Quadratic drag as described in this paper. (c) Linear drag as described in this paper. (d) Work done on the mean flow by propagating drag, (e) quadratic nonpropagating drag, and (f) linear nonpropagating drag. (g) Fraction of linear nonpropagating drag to total nonpropagating drag. (h) Fraction of nonpropagating drag to all drag and (i) nonpropagating work to all work.

need to worry about parameterizing this medium-scale nonpropagating drag.

In terms energy, the proposed nonpropagating drag from the medium-scale topography $\left(k u_{0}<f\right)$ leads to 2.7 TW of work against low-frequency flows (Figs. 13e,f). This is very substantial compared to $0.5 \mathrm{TW}$ from the short-scale lee waves $\left(k u_{0} / f<1\right)$ (Fig. 13d; Naveira Garabato et al. 2013). As pointed out by Trossman et al. (2013, 2016), there is nonpropagating drag for short-scale topography as well. They found that this short-scale nonpropagating drag in a $1 / 12^{\circ}$ model was similar to the radiating drag, so short-scale bathymetry potentially contributes $1 \mathrm{TW}$. Global tides are thought to contribute another 1 TW to the near-bottom internal wave field (Egbert and Ray 2003), so the $2.7 \mathrm{TW}$ proposed here is likely to be comparable to those sources.

A caveat against such a large loss of energy due to mediumscale topography is a lack of direct observational evidence. In the Southern Ocean, direct observations of dissipation and internal wave estimates of mixing have not seen enough dissipation to account for the energy lost from short-scale topography, let alone the larger values we are positing here (St. Laurent et al. 2012; Waterman et al. 2013). However, as noted by (Klymak 2018), the dissipation due to medium scale topography is very localized and in the lee of topographic features, in contrast to "lee wave" energy, which we would expect to be more evenly distributed. It will also tend to not have a canonical internal wave ocean spectra or lead to the type of wave-wave interactions that are used to infer ocean dissipation rates from shear and strain fine structure (e.g., Gregg et al. 2003; Kunze et al. 2006). Given that the eddying flow changes direction, it is very hard for a regional observation program to capture these dynamics, and points to the need for more targeted observational process studies (e.g., Clément et al. 2016; Evans et al. 2020) and numerical ones.

If close to as large as predicted, this drag is likely important for open-ocean mixing. While the motions generated from mediumscale topography cannot propagate vertically $\left(k u_{0} / f<1\right)$, the turbulence from the breaking of internal waves above the topography creates a halo of dissipation and mixing with a vertical scale of $\pi u_{0} / N$ (Klymak 2018), which can reach scales of many hundreds of meters. This is similar to parameterizations of tidal mixing (Jayne and St. Laurent 2001; St. Laurent et al. 2002), due to approximately $1 \mathrm{TW}$ of input, which is typically distributed over approximately $300 \mathrm{~m}$ vertically, and due to $0.2-0.5 \mathrm{TW}$ of energy radiated from propagating lee waves (Nikurashin et al. 2014; Naveira Garabato et al. 2013) which is distributed over a similar vertical distance. Global circulation models with $1^{\circ}$ resolution have found significant effects on the global stratification and circulation when deep ocean mixing due to both tides and radiating lee waves sources have been included 
(Melet et al. 2013, 2014). Even if our crude 2.7 TW estimate of the dissipation due to nonlinear breaking at longer horizontal scales is an overestimate, it still seems likely to be an important source of deep ocean mixing worthy of further investigation.

Acknowledgments. Thank you to two anonymous reviewers whose insightful comments greatly improved the manuscript. This work was funded under the Office of Naval Research Flow Encountering Abrupt Topography Defense Research Initiative (Grant N00014-15-1-2585), and NSERC Discovery Grant 3279202006. Thanks to Odessa Murray who manages the High Performance Computing accounts for ONR. ACNG acknowledges the support of the Royal Society and the Wolfson Foundation. DB acknowledges support from NSF OCE 1756882.

\section{REFERENCES}

Abernathey, R., and P. Cessi, 2014: Topographic enhancement of eddy efficiency in baroclinic equilibration. J. Phys. Oceanogr., 44, 2107-2126, https://doi.org/10.1175/JPO-D-14-0014.1.

Aguilar, D., and B. Sutherland, 2006: Internal wave generation from rough topography. Phys. Fluids, 18, 066603, https:// doi.org/10.1063/1.2214538.

Bacmeister, J., and R. Pierrehumbert, 1988: On high-drag states of nonlinear stratified flow over an obstacle. J. Atmos. Sci., 45, 63-80, https://doi.org/10.1175/1520-0469(1988)045<0063: OHDSON $>2.0 . \mathrm{CO} ; 2$.

Bell, T. H., 1975: Topographically generated internal waves in the open ocean. J. Geophys. Res., 80, 320-327, https://doi.org/ 10.1029/JC080i003p00320.

Boyer, T., and Coauthors, 2013: World Ocean Database 2013. NOAA Atlas NESDIS 72, 208 pp.

Broadbridge, M. B., A. C. Naveira Garabato, and A. J. G. Nurser, 2016: Forcing of the overturning circulation across a circumpolar channel by internal wave breaking. J. Geophys. Res. Oceans, 121, 5436-5451, https://doi.org/10.1002/2015JC011597.

Buijsman, M. C., and Coauthors, 2014: Three-dimensional doubleridge internal tide resonance in Luzon Strait. J. Phys. Oceanogr., 44, 850-869, https://doi.org/10.1175/JPO-D-13-024.1.

Clément, L., E. Frajka-Williams, K. L. Sheen, J. A. Brearley, and A. C. N. Garabato, 2016: Generation of internal waves by eddies impinging on the western boundary of the North Atlantic. J. Phys. Oceanogr., 46, 1067-1079, https://doi.org/ 10.1175/JPO-D-14-0241.1.

de Lavergne, C., G. Madec, F. Roquet, R. M. Holmes, and T. J. McDougall, 2017: Abyssal ocean overturning shaped by seafloor distribution. Nature, 551, 181-186, https://doi.org/10.1038/ nature 24472.

Egbert, G. D., and R. D. Ray, 2003: Semi-diurnal and diurnal tidal dissipation from TOPEX/Poseidon altimetry. Geophys. Res. Lett., 30, 1907, https://doi.org/10.1029/2003GL017676.

Evans, D. G., E. Frajka-Williams, A. C. N. Garabato, K. L. Polzin, and A. Forryan, 2020: Mesoscale eddy dissipation by a "zoo" of submesoscale processes at a western boundary. J. Geophys. Res. Oceans, 125, e2020JC016246, https://doi.org/ 10.1029/2020JC016246.

Garner, S. T., 2005: A topographic drag closure built on an analytical base flux. J. Atmos. Sci., 62, 2302-2315, https://doi.org/ 10.1175/JAS3496.1.

Gent, P. R., and J. C. McWilliams, 1990: Isopycnal mixing in ocean circulation models. J. Phys. Oceanogr., 20, 150-155, https://doi.org/10.1175/1520-0485(1990)020<0150:IMIOCM> 2.0.CO;2.
Goff, J. A., and B. K. Arbic, 2010: Global prediction of abyssal hill roughness statistics for use in ocean models from digital maps of paleo-spreading rate, paleo-ridge orientation, and sediment thickness. Ocean Modell., 32, 36-43, https://doi.org/10.1016/ j.ocemod.2009.10.001.

Gregg, M. C., T. B. Sanford, and D. P. Winkel, 2003: Reduced mixing from the breaking of internal waves in equatorial waters. Nature, 422, 513-515, https://doi.org/10.1038/nature01507.

Jayne, S. R., and L. C. St. Laurent, 2001: Parameterizing tidal dissipation over rough topography. Geophys. Res. Lett., 28, 811-814, https://doi.org/10.1029/2000GL012044.

Klymak, J. M., 2018: Nonpropagating form drag and turbulence due to stratified flow over large-scale abyssal hill topography. J. Phys. Oceanogr., 48, 2383-2395, https://doi.org/10.1175/ JPO-D-17-0225.1.

—_, and S. M. Legg, 2010: A simple mixing scheme for models that resolve breaking internal waves. Ocean Modell., 33, 224-234, https://doi.org/10.1016/j.ocemod.2010.02.005.

_ S. Legg, and R. Pinkel, 2010: High-mode stationary waves in stratified flow over large obstacles. J. Fluid Mech., 644, 321-336, https://doi.org/10.1017/S0022112009992503.

- H. L. Simmons, D. Braznikov, S. Kelly, J. A. MacKinnon, M. H. Alford, R. Pinkel, and J. D. Nash, 2016: Reflection of linear internal tides from realistic topography: The Tasman continental slope. J. Phys. Oceanogr., 46, 3321-3337, https:// doi.org/10.1175/JPO-D-16-0061.1.

Kunze, E., E. Firing, J. M. Hummon, T. K. Chereskin, and A. M. Thurnherr, 2006: Global abyssal mixing inferred from lowered ADCP shear and CTD strain profiles. J. Phys. Oceanogr., 36, 1553-1576, https://doi.org/10.1175/JPO2926.1.

Lott, F., and M. J. Miller, 1997: A new subgrid-scale orographic drag parametrization: Its formulation and testing. Quart. J. Roy. Meteor. Soc., 123, 101-127, https://doi.org/10.1002/ qj.49712353704.

Marshall, D. P., M. H. P. Ambaum, J. R. Maddison, D. R. Munday, and L. Novak, 2017: Eddy saturation and frictional control of the Antarctic Circumpolar Current. Geophys. Res. Lett., 44, 286-292, https://doi.org/10.1002/2016GL071702.

Marshall, J., A. Adcroft, C. Hill, L. Perelman, and C. Heisey, 1997: A finite-volume, incompressible Navier-Stokes model for studies of the ocean on parallel computers. J. Geophys. Res., 102, 5753-5766, https://doi.org/10.1029/96JC02775.

Melet, A., R. Hallberg, S. Legg, and K. Polzin, 2013: Sensitivity of the ocean state to the vertical distribution of internal-tidedriven mixing. J. Phys. Oceanogr., 43, 602-615, https://doi.org/ 10.1175/JPO-D-12-055.1.

,,,--- and M. Nikurashin, 2014: Sensitivity of the ocean state to lee wave-driven mixing. J. Phys. Oceanogr., 44, 900-921, https://doi.org/10.1175/JPO-D-13-072.1.

Munday, D. R., H. L. Johnson, and D. P. Marshall, 2015: The role of ocean gateways in the dynamics and sensitivity to wind stress of the early Antarctic circumpolar current. Paleoceanography, 30, 284-302, https://doi.org/10.1002/2014PA002675.

Naveira Garabato, A. C., A. J. G. Nurser, R. B. Scott, and J. A. Goff, 2013: The impact of small-scale topography on the dynamical balance of the ocean. J. Phys. Oceanogr., 43, 647-668, https://doi.org/10.1175/JPO-D-12-056.1.

Nikurashin, M., and R. Ferrari, 2010: Radiation and dissipation of internal waves generated by geostrophic motions impinging on small-scale topography: Theory. J. Phys. Oceanogr., 40, 1055-1074, https://doi.org/10.1175/2009JPO4199.1.

$\_, \ldots$, N. Grisouard, and K. Polzin, 2014: The impact of finiteamplitude bottom topography on internal wave generation in 
the Southern Ocean. J. Phys. Oceanogr., 44, 2938-2950, https://doi.org/10.1175/JPO-D-13-0201.1.

Scinocca, J. F., and N. A. McFarlane, 2000: The parametrization of drag induced by stratified flow over anisotropic orography. Quart. J. Roy. Meteor. Soc., 126, 2353-2393, https://doi.org/ 10.1002/qj.49712656802.

Shakespeare, C. J., and A. M. Hogg, 2017: The viscous lee wave problem and its implications for ocean modelling. Ocean Modell., 113, 22-29, https://doi.org/10.1016/j.ocemod.2017.03.006.

St. Laurent, L., H. L. Simmons, and S. Jayne, 2002: Estimating tidally driven mixing in the deep ocean. Geophys. Res. Lett., 29, 2106, https://doi.org/10.1029/2002GL015633.

, A. Naveira Garabato, J. Ledwell, A. Thurnherr, J. Toole, and A. Watson, 2012: Turbulence and diapycnal mixing in Drake Passage. J. Phys. Oceanogr., 42, 2143-2152, https://doi.org/ 10.1175/JPO-D-12-027.1.

Thompson, A. F., and A. C. Naveira Garabato, 2014: Equilibration of the Antarctic circumpolar current by standing meanders. J. Phys. Oceanogr., 44, 1811-1828, https://doi.org/10.1175/JPO-D-13-0163.1.

Tozer, B., D. T. Sandwell, W. H. F. Smith, C. Olson, J. R. Beale, and P. Wessel, 2019: Global bathymetry and topography at
15 arc sec: SRTM15+. Earth Space Sci., 6, 1847-1864, https:// doi.org/10.1029/2019EA000658.

Trossman, D. S., B. K. Arbic, S. T. Garner, J. A. Goff, S. R. Jayne, E. J. Metzger, and A. J. Wallcraft, 2013: Impact of parameterized lee wave drag on the energy budget of an eddying global ocean model. Ocean Modell., 72, 119-142, https:// doi.org/10.1016/j.ocemod.2013.08.006.

S. Waterman, K. L. Polzin, B. K. Arbic, S. T. Garner, A. C. Naveira-Garabato, and K. L. Sheen, 2015: Internal lee wave closures: Parameter sensitivity and comparison to observations. J. Geophys. Res. Oceans, 120, 7997-8019, https://doi.org/ 10.1002/2015JC010892.

—_, B. K. Arbic, J. G. Richman, S. T. Garner, S. R. Jayne, and A. J. Wallcraft, 2016: Impact of topographic internal lee wave drag on an eddying global ocean model. Ocean Modell., 97, 109-128, https://doi.org/10.1016/ j.ocemod.2015.10.013

Waterman, S., A. C. Naveira Garabato, and K. L. Polzin, 2013: Internal waves and turbulence in the Antarctic Circumpolar Current. J. Phys. Oceanogr., 43, 259-282, https://doi.org/ 10.1175/JPO-D-11-0194.1. 